Pacific Journal of Mathematic 


\title{
A NOTE ON THE OUTER GALOIS THEORY OF RINGS
}

\author{
H. F. KREIMER
}

Let $G$ be a finite group of automorphisms of a ring $B$, and let $A$ be the subring of $G$-invariant elements of $B$. Call $B$ an outer semi-Galois extension of $A$, if the centralizer of $A$ in $B$ is the center of $B$ and $B$ is a separable extension of $A$ (i.e., the $(B, B)$-bimodule homomorphism of $B \otimes_{A} B$ onto $B$, which is determined by the ring multiplication in $B$, splits). The principal result of this paper is more easily stated here under the additional hypothesis that $A$ is a direct summand of the right $A$-module $B$.

Theorem. If $B$ is an outer semi-Galois extension of a subring $A_{0}$ and $A_{0}$ is a direct summand of the right $A_{0}$-module $B$, then the following statements are equivalent for an intermediate ring $A$.

(1) $B$ is an outer semi-Galois extension of $A$ and $A$ is a direct summand of the right $A$-module $B$.

(2) $B$ is a projective Frobenius extension of $A$.

(3) $A$ is the subring of invariant elements of $B$ with respect to a finite group of automorphisms of $B$ (not necessarily a subgroup of $G$ ).

For outer Galois theory, this result is an improvement on the Galois theory for noncommutative rings presented by the author in [7] and by Y. Miyashita in [8], since the characterization of the intermediate ring in the Galois correspondence does not depend on the choice of $G$. If $B$ is a commutative ring, then essentially the same result (with a different proof) can be found also in a forthcoming paper, "Galois theory in rings with infinitely many idempotents", by O.Villamayor and D. Zelinsky.

A general Galois correspondence between subrings of a ring $B$ and subrings of the ring of endomorphisms of the additive group of $B$ is described in $\S 1$, and the Galois closure of a subring in $B$ is defined. These results are used to sharpen a theorem on Frobenius extensions, and the basic concepts of the Galois theory of rings are summarized. In $\S 2$, the concept of outer semi-Galois extension is introduced. The principal results of the paper are proved in $\S 3$.

1. Preliminaries. For the most part, the terminology and notation in [7] are followed throughout this paper. The most notable exception is that, whereas the image of an element $a$ under a mapping $\varphi$ was denoted by $a \varphi$ in [7], the more common notation $\varphi(\alpha)$ will be 
used in the sequel. In particular, ring will mean ring with identity element and subring of a ring will mean subring which contains the identity element of the ring.

Let $B$ be a ring and let $\mathfrak{B}$ be the ring of all endomorphisms of the additive group of $B$. The operations of left multiplication on $B$ by elements of $B$ form a subring of $\mathfrak{B}$, which is naturally isomorphic to $B$. Thus $\mathfrak{B}$ may be regarded as an extension of $B$ and $\mathfrak{B}$ supports the structure of a left $B$-module. If $A$ is a subring of $B$ and Hom $\left(B_{A}, B_{A}\right)$ denotes the ring of right $A$-module endomorphisms of $B$, then $\operatorname{Hom}\left(B_{A}, B_{A}\right)$ is both a subring and a left $B$-submodule of $\mathfrak{B}$. But, if $\mathfrak{U}$ is a subring of $\mathfrak{B}$, then $B$ is a left $\mathfrak{U}$-module; and, if $\mathfrak{U}$ is both a subring and a left $B$-submodule of $\mathfrak{B}$, then $\mathfrak{A}$ contains the ring of left multiplications on $B$ by elements of $B$ and the ring Hom $\left.{ }_{\mathfrak{X}} B, \mathfrak{X} B\right)$ of left $\mathfrak{U}$-module endomorphisms of $B$ must be the ring: $\bar{A}_{R}$ of right multiplications on $B$ by elements of some subring $\bar{A}$ of $B$.

Let $\mathfrak{i}=\operatorname{Hom}\left(B_{A}, B_{A}\right)$ for a subring $A$ of $B$. The subring $\bar{A}$ of $B$ such that Hom $(\mathfrak{A} B, \mathfrak{Y} B)=\bar{A}_{R}$ will be called the Galois closure of $A$ in $B$. Clearly $A \subseteq \bar{A}$ and $\bar{A}$ is the largest subring of $B$ such that $\operatorname{Hom}\left(B_{A}, B_{A}\right)=\operatorname{Hom}\left(B_{\bar{A}}, B_{\bar{A}}\right)$. If $A=\bar{A}, A$ will be said to be Galois closed in $B . \quad 2$ is naturally isomorphic to $\operatorname{Hom}\left(\left(B \otimes{ }_{A} B\right)_{B}, B_{L}\right)$, which is the dual of the right $B$-module $B \otimes{ }_{A} B$; and $\operatorname{Hom}\left({ }_{B} \mathfrak{A},{ }_{B} B\right)$ is naturally isomorphic to the second dual of $B \otimes{ }_{A} B$. Now suppose that $B$ is a finitely generated, projective right $A$-module. Then $B \otimes{ }_{A} B$ is a finitely generated, projective right $B$-module; $\mathfrak{U}$ is a finitely generated, projective left $B$-module; $\mathfrak{Y}$ is naturally isomorphic to $B \otimes{ }_{A} \mathrm{Hom}\left(B_{A}, A_{A}\right)$; and $\operatorname{Hom}\left({ }_{B} \mathfrak{A},{ }_{B} B\right)$ is naturally isomorphic to $B \otimes{ }_{1} B$. Since

$$
\operatorname{Hom}\left(B_{A}, A_{A}\right) \subseteq \operatorname{Hom}\left(B_{A}, \bar{A}_{A}\right)=\operatorname{Hom}\left(B_{\bar{A}}, \bar{A}_{\bar{A}}\right) \text {, }
$$

the natural homomorphism of $B \otimes_{\bar{A}} \operatorname{Hom}\left(B_{\bar{A}}, \bar{A}_{\bar{A}}\right)$ into $\operatorname{Hom}\left(B_{\bar{A}}, B_{\bar{A}}\right)=\mathfrak{Y}$ must be epic. Therefore $B$ is a finitely genereted, projective right $\bar{A}$-module and $\mathfrak{U}$ is naturally isomorphic to $B \otimes_{\bar{A}} \operatorname{Hom}\left(B_{\bar{A}}, \bar{A}_{\bar{A}}\right)$ by [1, proposition A.1]. Moreover $B \otimes_{A} B=B \otimes_{\bar{A}} B$.

The following proposition gives an application of the concept of Galois closure to the theory of (projective) Frobenius extensions [6].

Proposition 1.1. Let $A$ be a subring of a ring $B$ such that $B$ is a finitely generated, projective right $A$-module; let $\mathfrak{U}=\operatorname{Hom}\left(B_{A}, B_{A}\right)$; and let $\vec{A}$ be the Galois closure of $A$ in $B$.

(1) If $B$ is a Frobenius extension of $A$, then $\mathfrak{U}$ is a Frobenius extension of $B$ and $\operatorname{Hom}\left(B_{A}, A_{A}\right)=\operatorname{Hom}\left(B_{\bar{A}}, \bar{A}_{\bar{A}}\right)$.

(2) If $\mathfrak{A}$ is a Frobenius extension of $B$, then $B$ is a Frobenius extension of $\bar{A}$. 
Proof. According to the definition and Remark 1 in $[6, \S 1,2]$, $\mathfrak{A}$ is a Frobenius extension of $B$ if and only if there is an $(\mathfrak{A}, B)$ bimodule isomorphism of $\operatorname{Hom}\left({ }_{B} \mathfrak{A},{ }_{B} B\right)$ onto $\mathfrak{A}$. Since there is a natural isomorphism of $B \otimes{ }_{A} B$ onto $\operatorname{Hom}\left({ }_{B} \mathfrak{A},{ }_{B} B\right), \mathfrak{U}$ is a Frobenius extension of $B$ if and only if there is an ( $\mathfrak{A}, B$ )-bimodule isomorphism of $B \otimes{ }_{A} B$ onto $\mathfrak{A}$. Suppose $B$ is a Frobenius extension of $A$. Then there is an $(A, B)$-bimodule isomorphism of $B$ onto $\operatorname{Hom}\left(B_{A}, A_{A}\right)$ by Remark 1 of [6, §1.2]. Consequently, there is an ( $\mathfrak{A}, B)$-bimodule isomorphism of $B \otimes{ }_{A} B$ onto $B \otimes_{A} \operatorname{Hom}\left(B_{A}, A_{A}\right)$. But $B \otimes_{A} B=B \otimes_{\bar{A}} B$ and $B \otimes_{A} \operatorname{Hom}\left(B_{A}, B_{A}\right)$ is naturally isomorphic to $\mathfrak{A}$. Therefore there is an ( $\mathfrak{A}, B$ )-bimodule isomorphism of $B \otimes{ }_{\bar{A}} B$ onto $\mathfrak{A}$.

Now suppose that there is an (भI, $B$ )-bimodule isomorphism of $B \otimes{ }_{\bar{A}} B$ onto $\mathfrak{A}$, and let $\gamma \in \mathfrak{A}$ correspond to $1 \otimes 1 \in B \otimes{ }_{A} B$ under this isomorphism. If $b, b^{\prime} \in B$ and $\varphi \in \mathfrak{A}$; then $\varphi \circ\left(b^{\prime} \cdot \gamma \cdot b\right)=\varphi\left(b^{\prime}\right) \cdot \gamma \cdot b$, since both correspond to $\varphi\left(b^{\prime}\right) \otimes b$ under the given ( $\mathfrak{A}, B$ )-bimodule isomorphism of $B \otimes{ }_{A} B$ onto $\mathfrak{A}$. It follows readily from the definition of $\bar{A}$ that $\gamma \cdot b \in \operatorname{Hom}\left(B_{\bar{A}}, \bar{A}_{\bar{A}}\right)$. Also $\gamma \cdot a=a \cdot \gamma$ for $a \in \bar{A}$. There must exist a positive integer $n$ and elements $b_{j}, b_{j}^{\prime}$ of $B, 1 \leqq j \leqq n$, such that $\sum_{j=1}^{n} b_{j} \cdot \gamma \cdot b_{j}^{\prime}$ is the identity map on $B$. If $x \in B$ and $\psi \in \operatorname{Hom}\left(B_{\bar{A}}, \bar{A}_{\bar{A}}\right)$; then

$$
\psi(x)=\psi\left(\sum_{j=1}^{n} b_{j} \cdot \gamma\left(b_{j}^{\prime} \cdot x\right)\right)=\sum_{j=1}^{n} \psi\left(b_{j}\right) \cdot \gamma\left(b_{j}^{\prime} \cdot x\right)=\gamma\left(\left(\sum_{j=1}^{n} \psi\left(b_{j}\right) \cdot b_{j}^{\prime}\right) \cdot x\right) .
$$

Thus $\psi=\gamma \cdot c$ for $c=\sum_{j=1}^{n} \psi\left(b_{j}\right) \cdot b_{j}^{\prime}$. Therefore the composition of the $(\bar{A}, B)$-bimodule monomorphism of $B$ into $B \otimes_{\bar{A}} B$ which maps $b$ onto $1 \otimes b$ with the given $(\mathfrak{A}, B)$-bimodule isomorphism of $B \otimes{ }_{A} B$ onto $\mathfrak{A}$ is an $(\bar{A}, B)$-bimodule isomorphism of $B$ onto $\operatorname{Hom}\left(B_{\bar{A}}, \bar{A}_{\bar{A}}\right)$. Conse-

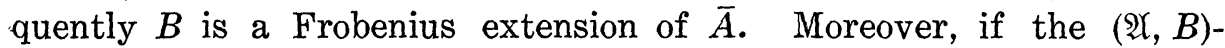
bimodule isomorphism of $B \otimes_{\bar{A}} B$ onto $\mathfrak{U}$ is derived from an $(A, B)$ bimodule isomorphism of $B$ onto $\operatorname{Hom}\left(B_{A}, A_{A}\right)$, then $\gamma \in \operatorname{Hom}\left(B_{A}, A_{A}\right)$. Therefore $\gamma \cdot b \in \operatorname{Hom}\left(B_{A}, A_{A}\right)$ for $b \in B$, and

$$
\operatorname{Hom}\left(B_{A}, A_{A}\right)=\operatorname{Hom}\left(B_{\bar{A}}, \bar{A}_{\bar{A}}\right) \text {. }
$$

Corollary 1.2. Let $A$ be a Galois closed subring of a ring $B$ such that $B$ is a finitely generated, projective right $A$-module. $B$ is a Frobenius extension of $A$ if, and only if, $\operatorname{Hom}\left(B_{A}, B_{A}\right)$ is a Frobenius extension of $B$.

The following three lemmas are restatements of results contained in [9].

Lemma 1.3. Let $A$ be a subring of $B$, let $\mathfrak{A}=\operatorname{Hom}\left(B_{A}, B_{A}\right)$, and let $\mathscr{T}\left(B_{A}\right)$ be the trace ideal of the right $A$-module $B . \quad \mathscr{T}\left(B_{A}\right)=A$ 
if, and only if, $B$ is a finitely generated, projective left $\mathfrak{A}$-module and $\operatorname{Hom}(\mathfrak{Q} B, \mathfrak{a} B)=A_{R}$.

Lemma 1.4. Let $\mathfrak{A}$ be a subring and left $B$-submodule of $\mathfrak{B}$, let $\bar{A}$ be that subring of $B$ such that $\operatorname{Hom}(\mathfrak{\Re} B, \mathfrak{A} B)=\bar{A}_{R}$, and let $\mathscr{T}\left({ }_{\mathfrak{A}} B\right)$ be the trace ideal of the left $\mathfrak{H}$-module $B . \mathscr{T}(\mathfrak{A} B)=\mathfrak{A}$ if, and only if, $B$ is a finitely generated, projective right $\bar{A}$-module and $\mathfrak{A}=$ $\operatorname{Hom}\left(B_{\bar{A}}, B_{\bar{A}}\right)$.

Lemma 1.5. Let $A$ be a subring of $B . \mathscr{T}\left(B_{A}\right)=A$ if, and only if, $A$ is a direct summand of the right $A$-module $B$.

In the application of these results, the following lemma is useful.

Lemma 1.6. Let $A$ be a subring of $B$ such that $B$ is a finitely generated, projective right $A$-module. $\mathscr{T}\left(B_{A}\right)=A$ if, and only if, $B$ is a faithfully flat right A-module.

Proof. Since $B$ is a projective right $A$-module, $B$ is a flat right $A$-module. Suppose $\mathscr{T}\left(B_{A}\right)=A$. Then $A$ is a direct summand of the right $A$-module $B$ by Lemma 1.5 , and $A \otimes_{A} X$ is a direct summand of the additive group $B \otimes{ }_{A} X$ for any unital left $A$-module $X$. But $A \otimes{ }_{A} X$ is naturally isomorphic to $X$. Consequently, if $B \otimes{ }_{A} X=$ 0 then $X=0$; and $B$ is a faithfully flat right $A$-module by [3, Chapter 1, §3, No. 1, Proposition 1].

Conversely, suppose $B$ is a faithfully flat right $A$-module. Let $\mathfrak{U}=\operatorname{Hom}\left(B_{A}, B_{A}\right)$ and let $\tau$ be the evaluation map of $\operatorname{Hom}\left(B_{A}, A_{A}\right) \otimes_{\mathfrak{X}} B$ into $A . \quad \mathfrak{U}$ is naturally isomorphic to $B \otimes_{A} \operatorname{Hom}\left(B_{A}, A_{A}\right), B \otimes_{A} A$ is naturally isomorphic to $B$, and the map $1 \otimes \tau$ of $B \otimes_{A} \operatorname{Hom}\left(B_{A}, A_{A}\right) \otimes_{\mathfrak{A}}$ $B$ into $B \otimes_{A} A$ corresponds to the natural isomorphism of $\mathfrak{U} \otimes_{\mathfrak{A}} B$ onto $B$. Therfore $1 \otimes \tau$ is an isomorphism, and $\tau$ is an isomorphism by [3, Chapter $1, \S 3$, No. 1, Proposition 2]. Since $\mathscr{T}\left(B_{A}\right)$ is the image of $\tau, \mathscr{T}\left(B_{A}\right)=A$.

From Lemmas 1.3 and 1.4 one obtains a Jacobson-Bourbaki type of correspondence [cf.5] between the set of subrings $A$ of $B$ such that $B$ is a finitely generated, projective right $A$-module and $\mathscr{T}\left(B_{A}\right)=$ $A$, and the set of subrings $\mathfrak{A}$ of $\mathfrak{B}$ such that $\mathfrak{A}$ is a left $B$-submodule of $\mathfrak{B}, B$ is a finitely generated, projective left $\mathfrak{Q}$-module, and $\mathscr{T}\left({ }_{\mathfrak{A}} B\right)=$ A. Call $B$ a generalized Galois extension of a subring $A$ if $B$ is a finitely generated, projective right $A$-module and $\mathscr{T}\left(B_{A}\right)=A$. In the definition of generalized Galois extension $B$ of a subring $A$, the requirement that $\mathscr{T}\left(B_{A}\right)=A$ may be replaced by either of the equivalent conditions given in Lemmas 1.5 and 1.6. Lemma 1.3 asserts that $\mathscr{T}\left(B_{A}\right)=A$ is a sufficient condition for a subring $A$ of $B$ to be Galois 
closed in $B$. In particular, if $B$ is a generalized Galois extension of a subring $A$, then $A$ is Galois closed in $B$. It is a consequence of Corollary 1.2 that a generalized Galois extension $B$ of a subring $A$ is a Frobenius extension if, and only if, $\operatorname{Hom}\left(B_{A}, B_{A}\right)$ is a Frobenius extension of $B$. This assertion may be seen to be equivalent to the corollary in $[6, \S 2.4]$ by observing that, if the right $A$-module $B$ possesses a direct summand which is isomorphic to $A$, then there exists a right $A$-module homomorphism of $B$ onto $A$ and $\mathscr{T}\left(B_{A}\right)=A$.

Let $G$ be a finite group of automorphism of a ring $B$, let $A$ be the subring of $G$-invariant elements of $B$, and let $\Delta$ be the crossed product of $B$ and $G$ with trivial factor set. Clearly $A$ is Galois closed in $B$ and there is a canonical ring homomorphism $i$ of $\Delta$ into $\operatorname{Hom}\left(B_{A}, B_{A}\right)$.

Proposition 1.7. The following statements are equivalent.

(1) $G$ is a strongly independent group of automorphisms of $B$.

(2) There exist a positive integer $n$ and elements $x_{j}, y_{j}$ of $B$, $1 \leqq j \leqq n$, such that $\sum_{j=1}^{n} \sigma\left(x_{j}\right) \cdot y_{j}=\delta_{1, \sigma}$ for all $\sigma \in G$.

(3) $B$ is a finitely generated, projective right $A$-module and $i$ is an isomorphism of $\triangle$ onto $\operatorname{Hom}\left(B_{A}, B_{A}\right)$.

Proof. If $\sum_{j=1}^{n} \sigma\left(x_{j}\right) \cdot y_{j}=\delta_{1, \sigma}$ for all $\sigma \in G$, then $\sum_{j=1}^{n} \sigma\left(x_{j}\right) \cdot \tau\left(y_{j}\right)=$ $\tau\left(\sum_{j=1}^{n} \tau^{-1} \sigma\left(x_{j}\right) \cdot y_{j}\right)=\delta_{\sigma, \tau}$ for all $\sigma, \tau \in G$. Therefore, it is a consequence of [7, Proposition 2.3] that $G$ is a strongly independent set of automorphisms of $B$ if, and only if, there exists a positive integer $n$ and elements $x_{j}, y_{j}$ of $B, 1 \leqq j \leqq n$, such that $\sum_{j=1}^{n} \sigma\left(x_{j}\right) \cdot y_{j}=\delta_{1, \sigma}$ for all $\sigma \in G$. The equivalence of statements 2 and 3 is proved in [4, Th. 1].

Following the terminology in [2], call $B$ a Galois extension of $A$ relative to $G$ if any of the statements of Proposition 1.7 is satisfied. Call $B$ an outer Galois extension of $A$ if $B$ is a Galois extension of $A$ and the centralizer of $A$ in $B$ is the center of $B$. Now suppose $B$ is a Galois extension of $A$ relative to $G$, and let $\mathfrak{A}=\operatorname{Hom}\left(B_{A}, B_{A}\right)$. Then $G$ freely generates the left $B$-module $\mathfrak{A}$. Let $\left\{\sigma^{*} \mid \sigma \in G\right\}$ be the dual basis for the right $B$-module $\operatorname{Hom}\left({ }_{B} \mathfrak{A},{ }_{B} B\right)$. But Hom $\left({ }_{B} \mathfrak{A},{ }_{B} B\right)$ is also a left $\mathfrak{X}$-module; and, for $b \in B$ and $\rho, \sigma \in G, \rho \cdot \sigma^{*}=\left(\sigma \cdot \rho^{-1}\right)^{*}$ and $b \cdot \sigma^{*}=\sigma^{*} \cdot \sigma(b)$. It is easily verified that the left $B$-module homomorphism of $\mathfrak{U}$ into $\operatorname{Hom}\left({ }_{B} \mathfrak{A},{ }_{B} B\right)$ which maps $\sigma$ onto $\left(\sigma^{-1}\right)^{*}$, for $\sigma \in G$, is an ( $\mathfrak{A}, B)$-bimodule isomorphism. Therefore $\mathfrak{A}$ is a Frobenius extension of $B . \quad B$ is a Frobenius extension of $A$ by Corollary 1.2. Let $n$ be a positive integer and let $x_{j}, y_{j}$ be elements of $B$ for $1 \leqq j \leqq n$, such that $\sum_{j=1}^{n} x_{j} \otimes y_{j} \in B \otimes \otimes_{A} B$ corresponds to $1^{*} \in \operatorname{Hom}\left({ }_{B} \mathfrak{A},{ }_{B} B\right)$ under the natural isomorphism of $\operatorname{Hom}\left({ }_{B} \mathfrak{A},{ }_{B} B\right)$ onto $B \otimes_{A} B$. Then

$$
\sum_{j=1}^{n} x_{j} \cdot y_{j}=1 \text { and } \sum_{j=1}^{n} b x_{j} \otimes y_{j}=\sum_{j=1}^{n} x_{j} \otimes y_{j} b
$$


for every $b \in B$. Thus $B$ is also a separable extension of $A$.

Proposition 1.8. Let $B$ be a Galois extension of $A$ with Galois group $G$. The following statements are equivalent.

(1) $A$ is a direct summand of the right $A$-module $B$.

(2) $B$ is a faithfully flat right A-module.

(3) $\mathscr{T}\left(B_{A}\right)=A$.

(4) There exists $c \in B$ such that $\sum_{\sigma \in G} \sigma(c)=1$.

Proof. Statements 1, 2, and 3 are equivalent by Lemmas 1.5 and 1.6. Also, statement 4 implies statement 1 by [7, Lemma 2.8]. Now suppose that $B$ is a faithfully flat right $A$-module and let $\omega=\sum_{\sigma \in G} \sigma$. $1 \otimes \omega$ is a left $B$-module homomorphism of $B \otimes_{A} B$ into $B \otimes_{A} A$, and $B \otimes_{A} A$ is naturally isomorphic to $B$. There exist a positive integer $n$ and elements $x_{j}, y_{j}$ of $B, 1 \leqq j \leqq n$, such that $\sum_{j=1}^{n} \sigma\left(x_{j}\right) \cdot y=\delta_{1, \sigma}$ for $\sigma \in G$. But then $\sum_{j=1}^{n} x_{j} \cdot \omega\left(y_{j}\right)=1$; and, consequently, $1 \otimes \omega$ is an epimorphism. Since $B$ is a faithfully flat right $A$-module, $\omega$ is an epimorphism and there must exist $c \in B$ such that $\omega(c)=1$. Therefore statement 2 implies statement 4 .

It follows from [7, Corollary 3.7 and Lemmas 3.2 and 2.8] that $B$ is a $K$-ring with respect to $G$ if, and only if, $B$ is a Galois extension of $A$ relative to $G$ and there exists $c \in B$ such that $\sum_{c \in G} \sigma(c)=$ 1. Propositions 1.7 and 1.8 may be used to formulate a number of conditions equivalent to $B$ being a $K$-ring with respect to $G$. In particular, $B$ is a $K$-ring with respect to $G$ if, and only if, $B$ is a generalized Galois extension of $A$ and $i$ is an isomorphism of $A$ onto $\operatorname{Hom}\left(B_{A}, B_{A}\right)$.

The preceding considerations are simpler in the case of commutative rings. For instance, suppose $A$ is a commutative subring of a ring $B$ such that $B$ is a finitely generated, projective right $A$-module. Then $\mathscr{T}\left(B_{A}\right)=A$ by [1, proposition A.3], and so $B$ is a generalized Galois extension of $A$. The situation for noncommutative rings is illustrated by the following example.

EXAMPLE 1.9 Let $B$ be the ring of $3 \times 3$ matrices over a field $F$ of characteristic two; and let $e_{i j}$ denote the element of $B$ with entry 1 in the $i$-th row and $j$-th column and entry 0 elsewhere, for $1 \leqq i$, $j \leqq 3$. Let $\sigma$ be the inner automorphism of $B$ determined by $e_{12}+e_{21}+e_{33}$. Then

$$
\sigma\left[\begin{array}{lll}
a_{11} & a_{12} & a_{13} \\
a_{21} & a_{22} & a_{23} \\
a_{31} & a_{32} & a_{33}
\end{array}\right]=\left[\begin{array}{lll}
a_{22} & a_{21} & a_{23} \\
a_{12} & a_{11} & a_{13} \\
a_{32} & a_{31} & a_{33}
\end{array}\right] \text { for } a_{i j} \in F, \quad 1 \leqq i, j \leqq 3 .
$$


$\sigma$ generates a subgroup $G$ of order two in the group of all automorphisms of $B$. Let $A$ be the subring of $G$-invariant elements of $B$. Since statement 2 of Proposition 1.7 is satisfied for $x_{1}=e_{11}=y_{1}$, $x_{2}=e_{22}=y_{2}, x_{3}=e_{32}$, and $y_{3}=e_{23} ; B$ is a Galois extension of $A$ relative to $G$. But since the characteristic of $F$ is two,

$$
(1+\sigma)\left[\begin{array}{lll}
a_{11} & a_{12} & a_{13} \\
a_{21} & a_{22} & a_{23} \\
a_{31} & a_{32} & a_{33}
\end{array}\right]=\left[\begin{array}{ccc}
a_{11}+a_{22} & a_{12}+a_{21} & a_{13}+a_{23} \\
a_{12}+a_{21} & a_{11}+a_{22} & a_{13}+a_{23} \\
a_{31}+a_{32} & a_{31}+a_{32} & 0
\end{array}\right]
$$

for $a_{i j} \in F, 1 \leqq i, j \leqq 3$. Therefore there is no element $c$ of $B$ such that $(1+\sigma)(c)$ is the identity matrix and $B$ is not a $K$-ring with respect to $G$. In particular, $B$ is a finitely generated, projective right $A$-module and $A$ is Galois closed in $B$, but $B$ is not a generalized Galois extension of $A$ [cf. 9, Remark 3]. Moreover, since $B$ is a Frobenius extension of $A$, this example demonstrates that Corollary 1.2 is a sharper result than the corollary in $[6, \S 2.4]$.

2. Outer semi-Galois extensions. The central idempotent elements of a ring play an important role in the outer Galois theory of rings, as the following lemma indicates.

LEMMA 2.1. Let $A$ be a subring of a ring $B$, such that the centralizer of $A$ in $B$ is the center of $B$ and the left $B$-module Hom $\left(B_{A}, B_{A}\right)$ is freely generated by a finite set $M$ of automorphisms of $B$ over $A$. $\eta \in \operatorname{Hom}\left(B_{A}, B_{A}\right)$ is a ring endomorphism such that $\eta(1)=1$ if, and only if, $\eta=\sum_{\sigma \in M} e_{\sigma} \cdot \sigma$ where $\left\{e_{\sigma} \mid \sigma \in M\right\}$ is a set of pairwise orthogonal, central idempotents in $B$ such that $\sum_{\sigma \in M} e_{\sigma}=1$.

Proof. Let $\eta \in \operatorname{Hom}\left(B_{A}, B_{A}\right)$; since the left $B$-module Hom $\left(B_{A}, B_{A}\right)$ is freely generated by $M, \eta$ has a unique representation as $\eta=\sum_{\sigma \in M} e_{\sigma} \cdot \sigma$ where $e_{\sigma} \in B$ for $\sigma \in M$. Suppose $\eta$ is a ring endomorphism of $B$ such that $\eta(1)=1$. Then $\sum_{\sigma \in M} e_{\sigma}=\eta(1)=1$, and $\eta(x) \cdot \sum_{\sigma \in M} e_{\sigma} \cdot \sigma(y)=\eta(x)$. $\eta(y)=\eta(x y)=\sum_{\sigma \in M} e_{\sigma} \cdot \sigma(x) \cdot \sigma(y)$ for $x, y \in B$. Therefore $\sum_{\sigma \in M} \eta(x) \cdot e_{\sigma} \cdot$ $\sigma=\sum_{\sigma \in M} e_{\sigma} \cdot \sigma(x) \cdot \sigma$ and $\eta(x) \cdot e_{\sigma}=e_{\sigma} \cdot \sigma(x)$ for $x \in B$ and $\sigma \in M$. Since $\eta, \sigma \in \operatorname{Hom}\left(B_{A}, B_{A}\right) ; e_{\sigma}$ must be an element of the centralizer of $A$ in $B$, which is the center of $B$, for $\sigma \in M$. But then $e_{\sigma} \cdot \sigma=e_{\sigma} \cdot \eta=$ $\sum_{\tau \in M} e_{\sigma} \cdot e_{\tau} \cdot \tau$ and $e_{\sigma} \cdot e_{\tau}=\delta_{\sigma, \tau} \cdot e_{\sigma}$ for $\sigma, \tau \in M$. Thus $\left\{e_{\sigma} \mid \sigma \in M\right\}$ is a set of pairwise orthogonal, central idempotents in $B$ such that $\sum_{\sigma \in M} e_{\sigma}=$ 1. Conversely, suppose $\left\{e_{\sigma} \mid \sigma \in M\right\}$ is a set of pairwise orthogonal, central idempotents in $B$ such that $\sum_{\sigma \in M} e_{\sigma}=1$. Then $\eta(1)=\sum_{\sigma \in M} e_{\sigma}=$ 1 , and $\eta(x y)=\sum_{\sigma \in M} e_{\sigma} \cdot \sigma(x) \cdot \sigma(y)=\eta(x) \cdot \eta(y)$ for $x, y \in B$. Therefore $\eta$ is a ring endomorphism such that $\eta(1)=1$.

Let $E$ be the set of all central idempotent elements of a ring $B$, and partially order $E$ by setting $e \leqq f$ if $e \cdot f=e$ for $e, f \in E . \quad E$ is 
a Boolean algebra in which the intersection $e \cap f$ is $e \cdot f$, the union $e \cup f$ is $e+f-e \cdot f$, and the complement of $e$ is $1-e$, for $e, f \in E$. An automorphism of $B$ restricts to an automorphism of the Boolean algebra $E$; and, thereby, any group of automorphisms of $B$ is represented as a group of automorphisms of the Boolean algebra $E$.

Let $A$ be a subring of a ring $B$; and let $S$ be a finite set of pairwise orthogonal, central idempotents in $B$, such that $\sum_{e \in S} e=1$. The right $A$-module $B$ is a direct sum of its submodules $B e, e \in S$; and $B e$ is a ring containing $A e$ as a subring for each $e \in S$. Now assume that $S \subseteq A$. If $Y$ is a right $A$-module then $\operatorname{Hom}\left(B_{A}, Y_{A}\right)=$ $\Pi_{e \in S} \operatorname{Hom}\left(B e_{A}, Y_{A}\right)=\prod_{e \in S} \operatorname{Hom}\left(B e_{A e}, Y e_{A e}\right)$; and it is easily verified that $B$ is a finitely generated, projective right $A$-module if, and only if, $B e$ is a finitely generated, projective right $A e$-module for each $e \in S$. Likewise, it is easily verified that $A$ is a direct summand of the right $A$-module $B, B$ is a Frobenius extension of $A, A$ is Galois closed in $B$, the centralizer of $A$ in $B$ is the center of $B$, or $B$ is a separable extension of $A$, if and only if the respective condition is satisfied by the ring $B e$ and its subring $A e$ for each $e \in S$. Moreover the group of all automorphisms of $B$ over $A$ is canonically isomorphic to the direct product of the groups of automorphisms of $B e$ over $A e$, $e \in S$; and in the sequel it will be convenient to use this isomorphism to identify any group of automorphisms of $B$ e over $A e, e \in S$, with a subgroup of the group of automorphisms of $B$ over $A$.

LEMMA 2.2. Let $T$ be a finite set of pairwise orthogonal, central idempotents in a ring $B$, such that $\sum_{e \in T} e=1$; let $g$ be a groupoid of ring isomorphisms between elements of the set $\{B e \mid e \in T\}$; let $g\left(B e, B e^{\prime}\right)$ be the set of isomorphisms in $g$ which map $B e$ onto $B e^{\prime}$ for $e, e^{\prime} \in T$; and let $A=\left\{b \in B \mid \sigma(b e)=b e^{\prime}\right.$ for $\sigma \in g\left(B e, B e^{\prime}\right)$ and $e$, $\left.e^{\prime} \in T\right\}$. Then $A$ is a subring of $B$; and there exist a finite set $S$ of pairwise orthogonal, central idempotents in $B$, such that $\sum_{e \in S} e=1$ and $S \subseteq A$; and a group $G_{e}$ of automorphisms of Be for each $e \in S$, satisfying the following conditions.

(1) For each $e \in S, A e$ is the subring of $G_{e}$-invariant elements of $\mathrm{Be}$.

(2) If $g(B e, B e)$ is finite for each $e \in T$, then $G_{e}$ is finite for each $e \in S$. If in addition, for each $e \in T$, there exists $c \in B e$ such that $\sum_{\sigma \in g(B e, B e)} \sigma(c)=e$; then, for each $e \in S$, there exists $c \in B e$ such that $\sum_{\sigma \in G_{e}} \sigma(c)=e$.

(3) If $G(B e, B e)$ is a finite, strongly independent group of automorphisms of $B e$ for each $e \in T$, then $G_{e}$ is a finite, strongly independent group of automorphisms of Be for each $e \in S$.

Proof. The verification that $A$ is a subring of $B$ is straight- 
forward and will be omitted. The condition that $g\left(B e, B e^{\prime}\right)$ be nonempty for $e, e^{\prime} \in T$ defines an equivalence relation on $T$, and an equivalence class of elements of $T$ will be called a component of the groupoid $g$. Letting $e_{C}=\sum_{e \in C} e$ for each component $C$ of $g$, it is readily verified that $S=\left\{e_{C} \mid C\right.$ is a component of $\left.g\right\}$ is a finite set of pairwise orthogonal, central idempotents in $B$ such that $\sum_{e \in S} e=1$ and $S \subseteq A$. Let $C$ be a given component of $g$, let $m$ be the cardinality of $C$, let $e_{0}, e_{1}, \cdots, e_{m-1}$ be an enumeration of the distinct elements of $C$, and choose $\tau_{i} \in g\left(B e_{0}, B e_{i}\right)$ for $0 \leqq i \leqq m-1$. Observe that $g\left(B e_{i}, B e_{j}\right)=\tau_{j} \cdot g\left(B e_{0}, B e_{0}\right) \cdot \tau_{i}^{-1}$ for $0 \leqq i, j \leqq m-1$. It is convenient to define $\tau_{i}$ for every integer $i$ by requiring that $\tau_{i}=\tau_{j}$ if $i \equiv$ $j(\bmod m)$. Setting $\bar{\tau}\left(\sum_{i=0}^{m-1} b \cdot e_{i}\right)=\sum_{i=0}^{m-1} \tau_{i+1} \cdot \tau_{i}^{-1}\left(b \cdot e_{i}\right)$ for $b \in B, \bar{\tau}$ is an automorphism of order $m$ on the ring $B \cdot e_{C}$. Setting $\bar{\sigma}\left(\sum_{i=0}^{m-1} b \cdot e_{i}\right)=$ $\sum_{i=0}^{m-1} \tau_{i} \cdot \sigma \cdot \tau_{i}^{-1}\left(b \cdot e_{i}\right)$ for $b \in B$ and $\sigma \in g\left(B e_{0}, B e_{0}\right), \bar{\sigma}$ is an automorphism of the ring $B \cdot e_{C}$ and $\bar{\sigma} \cdot \bar{\tau}=\bar{\tau} \cdot \bar{\sigma}$. The correspondence of $\bar{\sigma}$ to $\sigma \in g\left(B e_{0}, B e_{0}\right)$ is a monomorphism of $g\left(B e_{0}, B e_{0}\right)$ into the group of automorphisms of $B \cdot e_{C}$; and, letting $G$ be the subgroup of the group of automorphisms of $B \cdot e_{C}$ which is generated by the image of $g\left(B e_{0}, B e_{0}\right)$ and $\bar{\tau}, G$ is the direct product of the image of $g\left(B e_{0}, B e_{0}\right)$ and the cyclic group of order $m$ generated by $\bar{\tau}$. Therefore $G$ is finite whenever $g\left(B e_{0}, B e_{0}\right)$ is finite. If $\rho \in g\left(B e_{i}, B e_{j}\right)$ for $0 \leqq i, j \leqq m-1$, then $\rho=\tau_{j} \cdot \sigma \cdot \tau_{i}^{-1}$ for some $\sigma \in g\left(B e_{0}, B e_{0}\right)$ and $\rho$ coincides with the restriction of $\bar{\tau}^{j-i} \cdot \bar{\sigma}$ to $B e_{i}$. Consequently, the subring of $G$-invariant elements of $B \cdot e_{C}$ is $A \cdot e_{C}$. Now assume that $g\left(B e_{0}, B e_{0}\right)$ is finite. If there exists $c \in B e_{0}$ such that $\sum_{\sigma \in g\left(B e_{0}, B e_{0}\right)} \sigma(c)=e_{0}$, then $c \in B \cdot e_{C}$ and $\sum_{i=0}^{m-1} \sum_{\sigma \in g\left(B e_{0}, B e_{0}\right)} \bar{\tau}^{i} \bar{\sigma}(c)=\sum_{i=0}^{m-1} \tau_{i}\left(e_{0}\right)=\sum_{i=0}^{m-1} e_{i}=e_{C}$. If $g\left(B e_{0}, B e_{0}\right)$ is a strongly independent group of automorphisms of $B e_{0}$, then there exist a positive integer $n$ and elements $x_{j}, y_{j}$ of $B e_{0}, 1 \leqq j \leqq n$, such that $\sum_{j=1}^{n} \sigma\left(x_{j}\right) \cdot y_{j}=\delta_{1, \sigma} \cdot e_{0}$ for $\sigma \in g\left(B e_{0}, B e_{0}\right)$. But then $\tau_{i}\left(x_{j}\right), \tau_{i}\left(y_{j}\right), 0 \leqq$ $i \leqq m-1$ and $1 \leqq j \leqq n$, are elements of $B \cdot e_{c}$; and, for any integer $k$ and $\sigma \in g\left(B e_{0}, B e_{0}\right), \sum_{i=0}^{m-1} \sum_{j=1}^{n} \bar{\tau}^{k} \bar{\sigma}\left(\tau_{i}\left(x_{j}\right)\right) \cdot \tau_{i}\left(y_{j}\right)=\sum_{i=0}^{m-1} \sum_{j=1}^{n} \tau_{i+k}\left(\sigma\left(x_{j}\right)\right)$. $\tau_{i}\left(y_{j}\right)$, which is $e_{C}$ if $k \equiv 0(\bmod m)$ and $\sigma=1$, but is 0 otherwise. Therefore $G$ is a strongly independent group of automorphisms of $B \cdot e_{C}$. To each e $\in S$, there corresponds a component of the groupoid $g$ and the preceding construction yields a group $G_{e}$ of automorphisms of $B \cdot e$, satisfying the requirements of the lemma.

The technique of working with a groupoid $g$ of ring isomorphisms, as in the preceding lemma, is due to Villamayor and Zelinsky [10]. Note that, if $A$ is a subring of a ring $B$ such that the centralizer of $A$ in $B$ is the center of $B$, then the center of $A$ is the intersection of $A$ with the center of $B$. The author is indebted to D. Zelinsky for suggesting the following theorem.

THEOREM 2.3. Let $A$ be a subring of a ring $B$ such that the 
centralizer of $A$ in $B$ is the center of $B$. The following statements are equivalent.

(1) $B$ is a separable extension of $A$ and $A$ is the subring of invariant elements of $B$ with respect to a finite group of automorphisms of $B$.

(2) There exists a finite set $S$ of pairwise orthogonal, central idempotents in $A$, such that $\sum_{e \in S} e=1$ and $B e$ is a Galois extension of $A e$ (relative to some finite group of automorphisms of $B e$ ) for each $e \in S$.

Proof. Suppose $B$ is a separable extension of $A$ and $A$ is the subring of invariant elements of $B$ with respect to a finite group $G$ of automorphisms of $B$. Since $B$ is a separable extension of $A$, there exist a positive integer $n$ and elements $x_{j}, y_{j}$ of $B, 1 \leqq j \leqq n$, such that $\sum_{j=1}^{n} x_{j} \cdot y_{j}=1$ and $\sum_{j=1}^{n} b x_{j} \otimes y_{j}=\sum_{j=1}^{n} x_{j} \otimes y_{j} b$ in $B \bigotimes_{A} B$ for every $b \in B$. Setting $e_{a}=\sum_{j=1}^{n} \sigma\left(x_{j}\right) \cdot y_{j}$ for $\sigma \in G, \sigma(b) \cdot e_{\sigma}=e_{\sigma} \cdot b$ for $b \in B$ and $\sigma \in G$. Therefore $e_{o}$ is an element of the centralizer of $A$ in $B$, which is the center of $B$, for $\sigma \in G$. Moreover, $e_{\sigma}^{2}=\sum_{j=1}^{n} \sigma\left(x_{j}\right)$. $y_{j} \cdot e_{\sigma}=\sum_{j=1}^{n} \sigma\left(x_{j}\right) \cdot e_{\sigma} \cdot y_{j}=\sum_{j=1}^{n} e_{\sigma} \cdot x_{j} \cdot y_{j}=e_{\sigma} \quad$ for $\sigma \in G$. Therefore $\left\{\sigma\left(e_{\tau}\right) \mid \sigma, \tau \in G\right\}$ is a finintie set of central idempotents in $B$, which generates a finite, $G$-stable subalgebra $E_{0}$ of the Boolean algebra $E$ of all central idempotents in $B$. Let $T$ be the set of minimal elements of $E_{0}$. For $e \in T$ and $f \in E_{0}$, either $e f=e$ or $e f=0$; and it is easily verified that $T$ is a finite, $G$-stable set of pairwise orthogonal, central idempotents in $B$, such that $\sum_{e \in T} e=1$. A groupoid $g$ of ring isomorphisms between elements of the set $\{B e \mid e \in T\}$ is obtained by letting $g\left(B e, B e^{\prime}\right)$ be the set of isomorphisms of $B e$ onto $B e^{\prime}$ which are restrictions of elements of $G$ for $e, e^{\prime} \in T$. Since $A$ is the subring of $G$-invariant elements of $B, A=\left\{b \in B \mid \sigma(b e)=b e^{\prime}\right.$ for $\sigma \in g\left(B e, B e^{\prime}\right)$ and $\left.e, e^{\prime} \in T\right\}$. Since $G$ is finite, $g(B e, B e)$ is finite for each $e \in T$. For $e \in T$ and $\rho \in G, \sum_{j=1}^{n} \rho\left(x_{j}\right) \cdot y_{j} e=e_{\rho} \cdot e$. Either $e_{\rho} \cdot e=e$ or $e_{\rho} \cdot e=0$; but, if $e_{\rho} \cdot e=e$, then $\rho(b) \cdot e=\rho(b) \cdot e_{\rho} \cdot e=e_{\rho} \cdot b \cdot e=b \cdot e$ for $b \in B$. Consequently, $\sum_{j=1}^{n} \sigma\left(x_{j} e\right) \cdot y_{j} e=\delta_{1, \sigma} \cdot e$ for all $\sigma \in g(B e, B e)$; and $g(B e, B e)$ is a finite, strongly independent group of automorphisms of $B e$ for $e \in T$. By Lemma 2.2, there exist a finite set $S$ of pairwise orthogonal, central idempotents in $A$, such that $\sum_{e \in S} e=1$; and, for each $e \in S$, a finite, strongly independent group $G_{e}$ of automorphisms of $B e$, such that $A e$ is the subring of $G_{e}$-invariant elements of $B e$. Therefore $B e$ is a Galois extension of $A e$ relative to $G_{e}$ for each $e \in S$.

Conversely, suppose there exists a finite set $S$ of pairwise orthogonal, central idempotents in $A$, such that $\sum_{e \in S} e=1$ and $B e$ is a Galois extension of $A e$ relative to a finite group $G_{e}$ of automorphisms of $B e$ for each $e \in S$. Since $B e$ is a Galois extension of $A e, B e$ is a separable extension of $A e$ for $e \in S$. Therefore $B$ is a separable extension of 
$A$. Let $G$ be the subgroup of the group of all automorphisms of $B$ over $A$, which is generated by the $G_{e}, e \in S$. Clearly $A$ is the subring of $G$-invariant elements of $B$. But $G$ is the direct product of its subgroups $G_{e}$ and $G_{e}$ is a finite group for $e \in S$. Therefore $G$ is finite.

Definition 2.4. Let $A$ be a subring of a ring $B$ such that the centralizer of $A$ in $B$ is the center of $B$. Call $B$ an outer semi-Galois extension of $A$ if either statement of Theorem 2.3 is satisfied.

Suppose $B$ is an outer semi-Galois extension of a subring $A$; and let $S$ be a finite set of pairwise orthogonal, central idempotents in $A$, such that $\sum_{e \in S} e=1$ and $B e$ is a Galois extension of $A e$ relative to a finite group $G_{e}$ of automorphisms of $B e$ for each $e \in S$. Then for each $e \in S, B e$ is a Frobenius extension of $A e$ and $G_{e}$ freely generates the left $B e$-module Hom $\left(B e_{A e}, B e_{A e}\right)$. Therefore $B$ is a Frobenius extension of $A$. Moreover, if $G$ is the group of automorphisms of $B$ which is generated by the $G_{e}, e \in S$, then $G$ is finite and it is easily verified that $G$ generates the left $B$-module $\operatorname{Hom}\left(B_{A}, B_{A}\right)$.

Proposition 2.5. Let $B$ be an outer semi-Galois extension of a subring $A$. Any finite set of automorphisms of $B$ over $A$ generates a finite group of automorphisms of $B$.

Proof. Let $M$ be a finite set of automorphisms of $B$ over $A$. First suppose $B$ is a Galois extension of $A$ relative to a finite group $G$ of automorphisms of $B$. Then $G$ freely generates the left $B$-module Hom $\left(B_{A}, B_{A}\right)$; and any automorphism $\eta$ of $B$ over $A$ has a unique representation as $\eta=\sum_{\sigma \in G} e_{\eta, \sigma} \cdot \sigma$, where $\left\{e_{\eta, \sigma} \mid \sigma \in G\right\}$ is a set of pairwise orthogonal, central idempotents in $B$ such that $\sum_{\sigma \in G} e_{\eta, \sigma}=1$, by Lemma 2.1. $\left\{\sigma\left(e_{\eta, \tau}\right) \mid \sigma, \tau \in G\right.$ and either $\eta \in M$ or $\left.\eta^{-1} \in M\right\}$ is a finite set of central idempotents in $B$, which generates a finite subalgebra $E_{0}$ of the Boolean algebra $E$ of all central idempotents in $B$. Let $H$ be the group of automorphisms of $B$ generated by $M$. If $\theta \in H$, then it may be verified by straightforwad calculations that $e_{0, \sigma} \in E_{0}$ for $\sigma \in G$. Since $E_{0}$ is finite, $H$ must be finite. Now suppose $B$ is an outer semi-Galois extension of $A$; and let $S$ be a finite set of pairwise orthogonal, central idempotents in $A$, such that $\sum_{e \in S} e=1$ and $B e$ is a Galois extension of $A e$ for each $e \in S$. But, for each $e \in S$, a finite set of automorphisms of $B e$ over $A e$ is obtained by restricting the elements of $M$ to $B e$, and it has now been established that this finite set of automorphisms of $B e$ over $A e$ generates a finite group $H_{e}$ of automorphisms of $B e$. Let $H$ be the subgroup of the group of all automorphisms of $B$ over $A$ which is generated by the $H_{e}, e \in S$. Then $M \subseteq H$, and $H$ is a finite group since it is the direct product of its 
subgroups $H_{e}, e \in S$. Therefore the group of automorphisms of $B$ generated by $M$ must be finite.

Suppose $B$ is a Galois extension of a subring $A$ relative to a finite group $G$ of automorphisms of $B$, and $S$ is a finite set of pairwise orthogonal, central idempotents in $B$, such that $S \subseteq A$ and $\sum_{e \in S} e=$ 1. For $e \in S$, the canonical projection of the group of all automorphisms of $B$ over $A$ onto the group of automorphisms of $B e$ over $A e$ determines a representation of $G$ as a group of automorphisms of $B e$. Since $A$ is the subring of $G$-invariant elements of $B, A e$ must be the subring of $G$-invariant elements of $B e$. Let $n$ be a positive integer and $x_{j}, y_{j}$ be elements of $B$ for $1 \leqq j \leqq n$, such that $\sum_{j=1}^{n} \sigma\left(x_{j}\right)$. $y_{j}=\delta_{1, \sigma}$ for all $\sigma \in G$. Then $x_{j} e, y_{j} e$ are elements of $B e$ for $1 \leqq j \leqq n$, and $\sum_{j=1}^{n} \sigma\left(x_{j} e\right) \cdot y_{j} e=\delta_{1, \sigma} \cdot e$ for all $\sigma \in G$. Therefore only $1 \in G$ acts as the identity automorphism on $B e$, the representation of $G$ as a group of automorphisms of $B e$ is faithful, and $B e$ is a Galois extension of $A e$ relative to $G$. It is evident from this observation, that to construct an example of an outer semi-Galois extension which is not a Galois extension one needs only to take the direct product of two outer Galois extensions which cannot have isomorphic Galois groups.

EXAMPLE 2.6. Let $B$ be an outer Galois extension of a subring $A$ relative to a nontrivial group $G$ of automorphisms of $B$, and let $B \times B$ denote the direct product of $B$ with itself. A faithful representation of $G$ as a group of automorphisms of $B \times B$ is obtained by setting $\sigma\left(b, b^{\prime}\right)=\left(\sigma(b), \sigma\left(b^{\prime}\right)\right)$ for $\sigma \in G$ and $b, b^{\prime} \in B$; and it is not difficult to verify that $B \times B$ is an outer Galois extension of its subring $A \times A$ relative to $G$. Since $B$ is trivially an outer Galois extension of $B, B \times B$ is an outer semi-Galois extension of its subring $A \times B$. In particular, $B \times B$ is a Frobenius extension of $A \times B$. But $B \times B$ cannot be a Galois extension of $A \times B$.

\section{Outer Galois theory.}

Lemma 3.1. Let $B$ be an outer Galois extension of a subring $A_{0}$ relative to a finite group $G$ of automorphisms of $B$; and let $A$ be $a$ subring of $B$ such that $A_{0} \subseteq A$ and $B$ is a Frobenius extension of A.

(1) If $A$ is Galois closed in $B$, then $B$ is an outer semi-Galois extension of $A$.

(2) If $B$ is a $K$-ring with respect to $G$, then $B$ is a generalized Galois extension of $A$.

Proof. Since the centralizer of $A_{0}$ in $B$ is the center of $B$, the centralizer of $A$ in $B$ must be the center of $B$. Since $B$ is a Galois 
extension of $A_{0}$, the left $B$-module $\operatorname{Hom}\left(B_{A_{0}}, B_{A_{0}}\right)$ is freely generated by $G$. Finally, since $B$ is a Frobenius extension of $A, B$ is a finitely generated, projective right $A$-module and there is an $(A, B)$-bimodule isomorphism of $B$ onto $\operatorname{Hom}\left(B_{A}, A_{A}\right)$. Let $\gamma \in \operatorname{Hom}\left(B_{A}, A_{A}\right)$ correspond to $1 \in B$ under an $(A, B)$-bimodule isomorphism of $B$ onto Hom $\left(B_{A}, A_{A}\right)$. Since $\gamma \in \operatorname{Hom}\left(B_{A_{0}}, B_{A_{0}}\right), \gamma$ has a unique representation as $\gamma=\sum_{\sigma \in G} e_{\sigma} \cdot \sigma$ where $e_{\sigma} \in B$ for $\sigma \in G$. If $a \in A$; then $\sum_{\sigma \in G} a \cdot e_{\sigma} \cdot \sigma=a \cdot \gamma=\gamma \cdot a=$ $\sum_{\sigma \in G} e_{\sigma} \cdot \sigma(a) \cdot \sigma$, since both correspond to $a$ under the given $(A, B)$ bimodule isomorphism of $B$ onto $\operatorname{Hom}\left(B_{A}, A_{A}\right)$, and $a \cdot e_{\sigma}=e_{\sigma} \cdot \sigma(a)$ for $\sigma \in G$. Therefore $e_{\sigma}$ must be an element of the centralizer of $A_{0}$ in $B$, which is the center of $B$, for $\sigma \in G$. Since there is a natural isomorphism of $B \otimes_{A} \operatorname{Hom}\left(B_{A}, A_{A}\right)$ onto $\operatorname{Hom}\left(B_{A}, B_{A}\right)$, there must exist a positive integer $m$ and elements $b_{i}, b_{i}^{\prime}$ of $B, 1 \leqq i \leqq m$, such that $\sum_{i=1}^{m} b_{i} \cdot \gamma \cdot b_{i}^{\prime}=\sum_{\sigma \in G} \sum_{i=1}^{m} b_{i} \cdot e_{\sigma} \cdot \sigma\left(b_{i}^{\prime}\right) \cdot \sigma$ is the identity automorphism of $B$. But then $\sum_{i=1}^{m} b_{i} \cdot e_{\sigma} \cdot \sigma\left(b_{i}^{\prime}\right)=\delta_{1, \sigma}$ for $\sigma \in G$, and $e_{1}$ must be a unit in the center of $B$. Since an $(A, B)$-bimodule isomorphism of $B$ onto Hom $\left(B_{A}, A_{A}\right)$ is given also by the mapping $b \leadsto \gamma \cdot e_{1}^{-1} \cdot b, b \in B$; one may assume that an $(A, B)$-bimodule isomorphism of $B$ onto $\operatorname{Hom}\left(B_{A}, A_{A}\right)$ has been chosen so that $e_{1}=1$.

Let $n$ be a positive integer and $x_{j}, y_{j}$ be elements of $B, 1 \leqq j \leqq n$, such that $\sum_{j=1}^{n} \sigma\left(x_{j}\right) \cdot y_{j}=\delta_{1, \sigma}$ for all $\sigma \in G$. Then $e_{\rho} \cdot \rho=\sum_{j=1}^{n} \rho\left(x_{j}\right) \cdot \gamma \cdot y_{j}$ and $e_{\rho} \cdot \rho \in \operatorname{Hom}\left(B_{A}, B_{A}\right)$ for $\rho \in G$. Therefore $\sum_{\sigma \in G} e_{\rho} \cdot \rho\left(e_{\sigma}\right) \cdot \rho \sigma=$ $e_{\rho} \cdot \rho \cdot \gamma=e_{\rho} \cdot \rho(1) \cdot \gamma=e_{\rho} \cdot \gamma=\sum_{\sigma \in G} e_{\rho} \cdot e_{\sigma} \cdot \sigma$, and $e_{\rho} \cdot \rho\left(e_{\sigma}\right)=e_{\rho} \cdot e_{\rho \sigma}$ for $\rho$, $\sigma \in G$. In particular, $e_{\rho}=e_{\rho} \cdot \rho\left(e_{1}\right)=e_{\rho} \cdot e_{\rho}$ for $\rho \in G$. Consequently, $\left\{\sigma\left(e_{*}\right) \mid \sigma, \tau \in G\right\}$ is a finite set of central idempotents in $B$, which generates a finite, $G$-stable subalgebra $E_{0}$ of the Boolean algebra $E$ of all central idempotents in $B$. If $T$ is the set of minimal elements of $E_{0}$; then $T$ is a finite, $G$-stable set of pairwise orthogonal, central idempotents in $B$ such that $\sum_{e \in T} e=1$. Let $e \in T, b \in B$, and $\sigma \in G$. If $e_{\sigma} \cdot \sigma(e)=0$, then $e_{\sigma} \cdot \sigma(b e)=0$; but if $e_{\sigma} \cdot \sigma(e) \neq 0$, then $e_{\sigma} \cdot \sigma(e)=\sigma(e)$ and $e_{\sigma} \cdot \sigma(b e)=\sigma(b e)$. Observe that for $e \in T$ and $\sigma, \tau \in G$ such that $e_{\sigma} \cdot \sigma(\tau(e))=\sigma(\tau(e)), e_{\sigma \tau} \cdot \sigma \tau(e)=e_{\sigma \tau} \cdot e_{\sigma} \cdot \sigma \tau(e)=\sigma\left(e_{\tau}\right) \cdot e_{\sigma} \cdot \sigma \tau(e)=\sigma\left(e_{\tau} \cdot \tau(e)\right)$. Therefore, if in addition $e_{\tau} \cdot \tau(e)=\tau(e)$, then $e_{\sigma \tau} \cdot \sigma \tau(e)=\sigma \tau(e)$. But letting $\tau=\sigma^{-1}$, one obtains from the preceding observation that, if $e_{\sigma} \cdot \sigma\left(\sigma^{-1}(e)\right)=\sigma\left(\sigma^{-1}(e)\right)$, then $e_{1} \cdot e=\sigma\left(e_{\sigma^{-1}} \cdot \sigma^{-1}(e)\right)$ or $e_{\sigma^{-1}} \cdot \sigma^{-1}(e)=\sigma^{-1}(e)$ since $e_{1}=1$. With these facts it may be verified that a groupoid $g$ of ring isomorphisms between elements of the set $\{B e \mid e \in T\}$ is obtained by letting $g\left(B e, B e^{\prime}\right)$ be the set of isomorphisms of $B e$ onto $B e^{\prime}$ which are restrictions of elements $\sigma$ of $G$ satisfying $e_{\sigma} \cdot \sigma(e)=e^{\prime}$ for $e, e^{\prime} \in T$. Let $\bar{A}$ be the Galois closure of $A$ in $B$. Clearly, $\bar{A}=$ $\{b \in B \mid \gamma(x b)=\gamma(x) \cdot b$ for all $x \in B\}$. But $\gamma=\sum_{\sigma \in G} e_{\sigma} \cdot \sigma$ and $e_{\sigma} \cdot \sigma \in$ Hom $\left(B_{A}, B_{A}\right)$ for $\sigma \in G$. Therefore $\bar{A}=\left\{b \in B \mid e_{\sigma} \cdot \sigma(b)=e_{o} \cdot b\right.$ for $\left.\sigma \in G\right\}=$ $\left\{b \in B \mid \eta(b e)=b \cdot e^{\prime}\right.$ for $\eta \in\left(B e, B e^{\prime}\right)$ and $\left.e, e^{\prime} \in T\right\}$. 
Now let $e \in T$ and let $H$, be the subgroup of automorphisms in $G$ which restrict to elements of $g(B e, B e)$. Since $G$ is finite, $g(B e, B e)$ is finite. Since $\sum_{j=1}^{n} \sigma\left(x_{j} e\right) \cdot y_{j} e=\delta_{1, \sigma} \cdot e$ for all $\sigma \in G, g(B e, B e)$ must be a strongly independent group of automorphisms of $B e$. Moreover, only $1 \in H$ restricts to the identity automorphism of $B e$. Therefore distinct elements of $H$ restrict to distinct elements of $g(B e, B e)$. Suppose that $B$ is a $K$-ring with respect to $G$. Then there exists $c \in B$ such that $\sum_{\sigma \in G} \sigma(c)=1$. Let $p$ be the index of $H$ in $G$, let $\left\{\tau_{k} \mid 1 \leqq k \leqq p\right\}$ be a system of representatives of the left cosets of $H$ in $G$, and let $c^{\prime}=\sum_{k=1}^{p} \tau_{k}(c)$. Then $\sum_{\sigma \in H} \sigma\left(c^{\prime}\right)=1$ and $\sum_{\left.r_{i \in g(B e}, B e\right)} \eta\left(c^{\prime} e\right)=$ $e$. By Lemma (2.2) there exist a finite set $S$ of pairwise orthogonal, central idempotents in $\bar{A}$, such that $\sum_{e \in S} e=1$; and, for each $e \in S$, a group $G_{e}$ of automorphisms of $B e$ with the properties that $B e$ is a Galois extension of $\bar{A} e$ relative to $G_{e}$, and $B e$ is a $K$-ring with respect to $G_{e}$ if $B$ is a $K$-ring with respect to $G$. Therefore, if $A=$ $\bar{A}$, then $B$ is an outer semi-Galois extension of $A$. If $B$ is a $K$-ring with respect to $G$, then $B e$ is a generalized Galois extension of $\bar{A} e$ for each $e \in S$. But then $B$ is a generalized Galois extension of $\bar{A}$ and $\mathscr{T}\left(B_{\bar{A}}\right)=\bar{A}$. Since $\operatorname{Hom}\left(B_{A}, A_{A}\right)=\operatorname{Hom}\left(B_{\bar{A}}, \bar{A}_{\bar{A}}\right)$ by Proposition 1.1, $\mathscr{T}\left(B_{\bar{A}}\right)=\mathscr{T}\left(B_{A}\right) \subseteq A \subseteq \bar{A}$. Therefore $\mathscr{T}\left(B_{A}\right)=A$ and $B$ is a generalized Galois extension of $A$.

THEOREM 3.2. Let $B$ be an outer semi-Galois extension of a subring $A_{0}$, and let $A$ be a subring of $B$ such that $A_{0} \leqq A$. The following statements are equivalent:

(1) $B$ is an outer semi-Galois extension of $A$.

(2) $A$ is Galois closed in $B$ and $B$ is a Frobenius extension of A.

(3) $A$ is the subring of invariant elements of $B$ with respect to some finite group of automorphisms of $B$.

Proof. Since $B$ is an outer semi-Galois extension of $A_{0}, B$ is a separable extension of $A_{0}$. Therefore $B$ must be a separable extension of $A$, and the equivalence of statements 1 and 3 follows from Definition (2.4). The remarks following Definition (2.4) establish that statements 1 and 3 imply statement 2 . But suppose $A$ is Galois closed in $B$ and $B$ is a Frobenius extension of $A$; and let $S$ be a finite set of pairwise orthogonal, central idempotents in $A_{0}$, such that $\sum_{e \in S} e=1$ and $B e$ is a Galois extension of $A_{0} e$ for each $e \in S$. Then $B e$ is an outer Galois extension of $A_{0} e, A e$ is a Galois closed subring of $B e$ such that $A_{0} e \subseteq A e$, and $B e$ is a Frobenius extension of $A e$, for $e \in S$. By Lemma 3.1, $B e$ is an outer semi-Galois extension of $A e$ for $e \in S$. It follows easily that $B$ is an outer semi-Galois extension of $A$. Therefore statement 2 implies statement 1 . 
If, in addition to the hypotheses of Theorem $3.2, A_{0}$ is a direct summand of the right $A_{0}$-module $B$; then Theorem 3.2 may be modified to read as follows:

THEOREM 3.3. Let $B$ be an outer semi-Galois extension of a subring $A_{0}$ such that $A_{0}$ is a direct summand of the right $A_{0}$-module $B$; and let $A$ be a subring of $B$ such that $A_{0} \leqq A$. The following statements are equivalent:

(1) $B$ is an outer semi-Galois extension of $A$ such that $A$ is $a$ direct summand of the right $A$-module $B$.

(2) $B$ is a Frobenius extension of $A$.

(3) $A$ is the subring of invariant elements of $B$ with respect to some finite group of automorphisms of $B$.

Proof. Statement 1 implies statement 3 and statement 3 implies statement 2 by Theorem 3.2. Suppose $B$ is a Frobenius extension of $A$; and let $S$ be a finite set of pairwise orthogonal, central idempotents in $A_{0}$, such that $\sum_{e \in S} e=1$ and $B e$ is a Galois extension of $A_{0} e$ relative to a finite group $G_{e}$ of automorphisms of $B e$ for each $e \in S$. Let $e \in S$. Then $A_{0} e$ is a direct summand of the $A_{0} e$-module $B e$. Therefore $B e$ is not only an outer Galois extension of $A_{0} e$, but $B e$ is also a $K$-ring with respect to $G_{e}$. Moreover $A e$ is a subring of $B e$ such that $A_{0} e \subseteq A e$ and $B e$ is a Frobenius extension of $A e$. By Lemma $3.1, B e$ is a generalized Galois extension of $A e$. Therefore $B$ is a generalized Galois extension of $A$. In particular, $A$ is Galois closed in $B$ and $A$ is a direct summand of the right $A$-module $B$. It now follows from Theorem 3.2 that statement 2 implies statement 1 .

Observe that in Theorem 3.3, the condition that $A_{0}$ (resp. $A$ ) be a direct summand of the right $A_{0}$ (resp. $A$ )-module $B$ may be replaced by either of the equivalent conditions given in Lemmas 1.5 and 1.6. Also, in view of Proposition 2.5, the word "group" may be replaced by "set" in statement 3 of either Theorem 3.2 or 3.3 .

\section{REFERENCES}

1. M. Auslander and O. Goldman, Maximal orders, Trans. Amer. Math. Soc., 97 (1960), 1-24.

2. The Brauer group of a commutative ring, Trans. Amer. Math. Soc. 97 (1960), 367-409.

3. N. Bourbaki, Algèbre commutative, Hermann, Paris, 1961.

4. F. R. De Meyer, Some notes on the general Galois theory of rings, Osaka J. Math. $\mathbf{2}(1965), 117-127$.

5. N. Jacobson, Lectures in abstract algebra, Vol. 3, Theory of fields and Galois theory, Van Nostrand, Princeton, N. J., 1964.

6. F. Kasch, Projektive Frobenius-Erweiterung, S.-B. Heidelberger Akad. Wiss. MathNatur. Kl. 1960/1961, 87-109. 
7. H. F. Kreimer, A Galois theory for non-commutative rings, Trans. Amer. Math. Soc. 127 (1967), 29-41.

8. Y. Miyashita, Finite outer Galois theory of non-commutative rings, J. Fac. Sci. Hokkaido Univ. Ser. I, 19 (1966), 114-134.

9. T. Nakayama, On a generalized notion of Galois extensions of a ring, Osaka J. Math. 15 (1963), 11-23.

10. O.E. Villamayor and D. Zelinsky, Galois theory for rings with finitely many idempotents, Nagoya Math. J. 27 (1966), 721-731.

Received September 16, 1968. The author gratefully acknowledges support in his research from the Florida State University Research Council and the National Science Foundation under grants GP-7098 and GP-8424.

Florida State UNIVERSity 


\section{PACIFIC JOURNAL OF MATHEMATICS}

\section{EDITORS}

\author{
H. ROYDEN \\ Stanford University \\ Stanford, California \\ Richard Pierce \\ University of Washington \\ Seattle, Washington 98105
}

\author{
J. DugundJI \\ Department of Mathematics \\ University of Southern California \\ Los Angeles, California 90007 \\ BASIL GoRDON \\ University of California \\ Los Angeles, California 90024
}

\section{ASSOCIATE EDITORS}
E. F. BECKENBACH
B. H. Neumann
F. WOLF
K. YoSHIDA

\section{SUPPORTING INSTITUTIONS}

\author{
UNIVERSITY OF BRITISH COLUMBIA \\ CALIFORNIA INSTITUTE OF TECHNOLOGY \\ UNIVERSITY OF CALIFORNIA \\ MONTANA STATE UNIVERSITY \\ UNIVERSITY OF NEVADA \\ NEW MEXICO STATE UNIVERSITY \\ OREGON STATE UNIVERSITY \\ UNIVERSITY OF OREGON \\ OSAKA UNIVERSITY \\ UNIVERSITY OF SOUTHERN CALIFORNIA
}

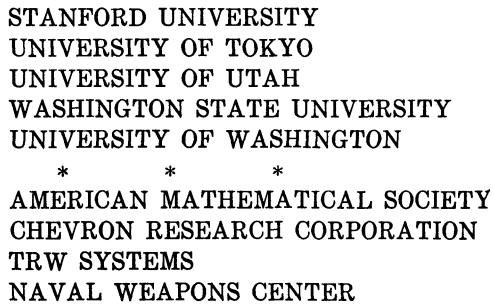

The Supporting Institutions listed above contribute to the cost of publication of this Journal, but they are not owners or publishers and have no responsibility for its content or policies.

Mathematical papers intended for publication in the Pacific Journal of Mathematics should be in typed form or offset-reproduced, double spaced with large margins. Underline Greek letters in red, German in green, and script in blue. The first paragraph or two must be capable of being used separately as a synopsis of the entire paper. It should not contain references to the bibliography. Manuscripts, in duplicate if possible, may be sent to any one of the four editors. Please classify according to the scheme of Math. Rev. 36, 1539-1546. All other communications to the editors should be addressed to the managing editor, Richard Arens, University of California, Los Angeles, California, 90024.

50 reprints are provided free for each article; additional copies may be obtained at cost in multiples of 50 .

The Pacific Journal of Mathematics is published monthly. Effective with Volume 16 the price per volume (3 numbers) is $\$ 8.00$; single issues, $\$ 3.00$. Special price for current issues to individual faculty members of supporting institutions and to individual members of the American Mathematical Society: $\$ 4.00$ per volume; single issues $\$ 1.50$. Back numbers are available.

Subscriptions, orders for back numbers, and changes of address should be sent to Pacific Journal of Mathematics, 103 Highland Boulevard, Berkeley, California, 94708.

PUBLISHED BY PACIFIC JOURNAL OF MATHEMATICS, A NON-PROFIT CORPORATION

Printed at Kokusai Bunken Insatsusha (International Academic Printing Co., Ltd.), 7-17, Fujimi 2-chome, Chiyoda-ku, Tokyo, Japan. 


\section{Pacific Journal of Mathematics}

\section{Vol. 31, No. $2 \quad$ December, 1969}

Efraim Pacillas Armendariz, Quasi-injective modules and stable torsion

classes..........................................

J. Adrian (John) Bondy, On Ulam's conjecture for separable graphs...

Vasily Cateforis and Francis Louis Sandomierski, On commutative rings over which the singular submodule is a direct summand for every module .....

Rafael Van Severen Chacon, Approximation of transformations with continuous

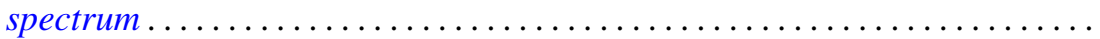

Raymond Frank Dickman and Alan Zame, Functionally compact spaces ...... 303

Ronald George Douglas and Walter Rudin, Approximation by inner

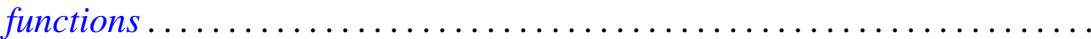

John Walter Duke, A note on the similarity of matrix and its conjugate

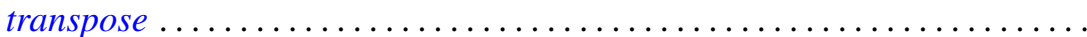

Micheal Neal Dyer and Allan John Sieradski, Coverings of mapping

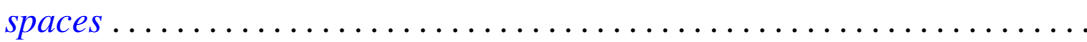

Donald Campbell Dykes, Weakly hypercentral subgroups of finite groups .....

Nancy Dykes, Mappings and realcompact spaces.....................

Edmund H. Feller and Richard Laham Gantos, Completely injective

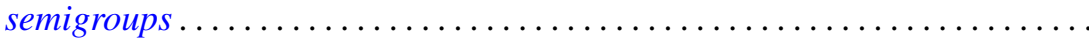

Irving Leonard Glicksberg, Semi-square-summable Fourier-Stieltjes

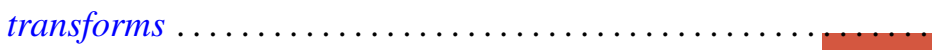

Samuel Irving Goldberg and Kentaro Yano, Integrability of almost cosymplectic structures...

Seymour Haber and Charles Freeman Osgood, On the sum $\sum\langle n \alpha\rangle^{-t}$ and numerical integration ..........................

Sav Roman Harasymiv, Dilations of rapidly decreasing functions ....

William Leonard Harkness and R. Shantaram, Convergence of a sequence of

transformations of distribution functions

Herbert Frederick Kreimer, Jr., A note on the outer Galois theory of rings ...

James Donald Kuelbs, Abstract Wiener spaces and applications to analysis. .

Roland Edwin Larson, Minimal $T_{0}$-spaces and minimal $T_{D}$-spaces...

A. Meir and Ambikeshwar Sharma, On Ilyeff's conjecture .

Isaac Namioka and Robert Ralph Phelps, Tensor products of compact convex sets....

James L. Rovnyak, On the theory of unbounded Toeplitz operators ....

Benjamin L. Schwartz, Infinite self-interchange graphs.......

George Szeto, On the Brauer splitting theorem...

Takayuki Tamura, Semigroups satisfying identity $x y=f(x$,

Kenneth Tolo, Factorizable semigroups .................. 